Check for updates

Cite this: J. Mater. Chem. A, 2020, 8 5467

Received 16th January 2020 Accepted 18th February 2020

DOI: $10.1039 / d 0 t a 00687 d$

\section{A flexible semitransparent photovoltaic supercapacitor based on water-processed MXene electrodes $\uparrow$}

\author{
Leiqiang Qin, $\mathbb{D} \ddagger^{\star a}$ Jianxia Jiang, $\dot{\dagger}^{\mathrm{ab}}$ Quanzheng Tao, ${ }^{\mathrm{a}}$ Chuanfei Wang, (D) ${ }^{c}$ \\ Ingemar Persson, ${ }^{a}$ Mats Fahlman, (iD ${ }^{c}$ Per O. Å. Persson, (D) a Lintao Hou, (D) ${ }^{b}$ \\ Johanna Rosen ${ }^{\star a}$ and Fengling Zhang (D) *ab
}

rsc.li/materials-a

Solar energy, although it has the highest power density available in terms of renewable energy, has the drawback of being erratic. Integrating an energy harvesting and storage device into photovoltaic energy storage modules is a viable route for obtaining self-powered energy systems. Herein, an MXene-based all-solution processed semitransparent flexible photovoltaic supercapacitor (PSC) was fabricated by integrating a flexible organic photovoltaic (OPV) with $\mathrm{Ti}_{3} \mathrm{C}_{2} \mathrm{~T}_{x}$ MXene as the electrode and transparent MXene supercapacitors with an organic ionogel as the electrolyte in the vertical direction, using $\mathrm{Ti}_{3} \mathrm{C}_{2} \mathrm{~T}_{x}$ thin film as a common electrode. In the quest for a semitransparent flexible PSC, $\mathrm{Ti}_{3} \mathrm{C}_{2} \mathrm{~T}_{x}$ MXene was first used as a transparent electrode for OPV with a high power conversion efficiency of $13.6 \%$. The ionogel electrolyte-based transparent MXene supercapacitor shows a high volumetric capacitance of $502 \mathrm{~F} \mathrm{~cm}^{-3}$ and excellent stability. Finally, a flexible PSC with a high average transmittance of over $33.5 \%$ was successfully constructed by allsolution processing and a remarkable storage efficiency of $88 \%$ was achieved. This strategy enables a simple route for fabricating MXene based high-performance all-solution-processed flexible PSCs, which is important for realizing flexible and printable electronics for future technologies.

\section{Introduction}

Various energy harvesting concepts are currently under investigation for the conversion of renewable sources into electrical energy, with the goal of providing long-term off-grid power for

${ }^{a}$ Department of Physics, Chemistry and Biology (IFM), Linköping University, Linköping, SE-58183, Sweden. E-mail: leiqiang.qin@liu.se; johanna.rosen@liu.se; fengling. zhang@liu.se

${ }^{b}$ Guangzhou Key Laboratory of Vacuum Coating Technologies and New Energy Materials, Physics Department, Jinan University, Guangzhou, 510632, PR China

${ }^{c}$ Laboratory of Organic Electronics, ITN, Linkoping University, SE-60174 Norrkoping, Sweden

$\dagger$ Electronic supplementary information (ESI) available. See DOI: 10.1039/d0ta00687d

\$ These authors contributed equally to this work. portable electronic devices and sensors. ${ }^{1-4}$ Organic photovoltaics (OPVs), as the most promising technology for long-term renewable energy production, have attracted enormous attention due to their great possibilities of high flexibility, light weight, low cost, and printing or roll-to-roll manufacturing..$^{5-8}$ However, PVs have a major drawback in their intermittent nature, i.e. the power output depends strongly on the fluctuation in light intensity caused by the diurnal cycle, weather, and season, among other factors, which results in an inability to maintain a constant and continuous electricity supply for electronic devices driven by PVs. ${ }^{9}$ Efficient energy storage devices are therefore needed as one potential solution to deal with fluctuating energy production. Therefore, integrating PVs with energy storage devices such as supercapacitors (SCs) offers a promising opportunity to store surplus available energy for later use during periods of non-generation or low power generation and to provide electricity reliably for long-term offgrid and commercial applications. ${ }^{\mathbf{1 0 - 1 2}}$

Recently, several attempts have been made to combine energy harvesting and storage into photovoltaic energy storage modules (PESM) for self-powered systems. ${ }^{\text {13-15 }}$ However, external circuits are commonly used as interconnections between the PVs and the charge storage part of the integrated devices, which results in low surface area utilization due to planar interconnection and is not compatible with roll-to-roll printing on flexible substrates. It is a challenge to explore devices with high mechanical flexibility and optical transparency to meet the needs of future ubiquitous electronics, including wearable devices and interactive systems. ${ }^{16,17}$ The ultimate objective of the field is to develop highly efficient, flexible, transparent, and low-cost PESMs in the vertical direction via printing or roll-to-roll manufacturing. ${ }^{18,19}$ Therefore, an all-solution-processed flexible PESM realized at low temperature is very suitable for implementation of upscaling and also for cost-effectiveness.

A commonly used transparent electrode in PV devices is indium tin oxide (ITO), which can offer high transmittance with low sheet resistance. However, ITO is mechanically brittle and 
experiences conductivity issues on plastic substrates. ${ }^{20,21}$ In addition, metal oxide films are commonly processed via vacuum sputtering at high temperatures, which is incompatible with printing and roll-to-roll manufacturing. Several emerging materials have been explored as transparent electrode materials, such as conductive polymers, graphene, carbon nanotubes, random networks of metal nanowires ( $\mathrm{Ag} / \mathrm{Cu} \mathrm{NWs})$, and hybrid films. In 2011, a new class of 2D materials emerged, made up of transition metal carbides/nitrides or carbonitrides. ${ }^{22}$ They were called MXenes, being of the general formula $\mathrm{M}_{n+1} \mathrm{X}_{n} \mathrm{~T}_{x}(n=1-3)$, where $\mathrm{M}$ represents an early transition metal, $\mathrm{X}$ is carbon and/or nitrogen, and $\mathrm{T}$ stands for the surface terminations $\mathrm{O}, \mathrm{OH}$, or $\mathrm{F} . \mathrm{Ti}_{3} \mathrm{C}_{2} \mathrm{~T}_{x}$ was the first MXene reported in 2011 and has been intensively studied. ${ }^{22-27} \mathrm{Ti}_{3} \mathrm{C}_{2} \mathrm{~T}_{x}$ possesses many attractive properties, including excellent electronic conductivity (up to $9880 \mathrm{~S} \mathrm{~cm}^{-1}$, surpassing other solutionprocessed 2D materials), ${ }^{28}$ high transmittance (transmitting $>97 \%$ of visible light per nanometer), ${ }^{29}$ and good flexibility. In addition, the hydrophilic surface of the $\mathrm{Ti}_{3} \mathrm{C}_{2} \mathrm{~T}_{x}$ MXene allows it to be processed with various solutions or inks, such as spin/ spray coating, blading, printing, and roll-to-roll manufacturing. ${ }^{30-32}$ Altogether, these interesting properties make $\mathrm{Ti}_{3} \mathrm{C}_{2} \mathrm{~T}_{x}$ MXene a promising candidate for several energy conversion and storage applications, including supercapacitors, batteries, electrocatalysis, and photocatalysis. Yet MXenes are comparatively unexplored in the field of optoelectronics.

Inspired by the electrical properties of MXenes, we have herein fabricated $\mathrm{Ti}_{3} \mathrm{C}_{2} \mathrm{~T}_{x}$ MXene-based all-solution-processed, semitransparent, and flexible solid-state photovoltaic supercapacitors (PSCs) via vertical stacking. Through spin-casting of colloidal solutions of $\mathrm{Ti}_{3} \mathrm{C}_{2} \mathrm{~T}_{x}$ nanosheets, highly conducting and transparent flexible films were obtained, made up of $\mathrm{Ti}_{3} \mathrm{C}_{2} \mathrm{~T}_{x}$ nanoflakes aligned parallel to the substrates. Consequently, the OPV models were constructed by solution processing at lower temperatures on the $\mathrm{Ti}_{3} \mathrm{C}_{2} \mathrm{~T}_{x}$ electrodes. To the best of our knowledge, this is the first report of pristine $\mathrm{Ti}_{3} \mathrm{C}_{2} \mathrm{~T}_{x}$ as transparent electrodes for solution-processed flexible OPVs. Assisted by the transfer-printing method, the transparent $\mathrm{Ti}_{3} \mathrm{C}_{2} \mathrm{~T}_{x}$ films were used as both the top electrode of the OPVs and the bottom electrode of the energy storage units. Finally, the transparent electrolyte layer and the other $\mathrm{Ti}_{3} \mathrm{C}_{2} \mathrm{~T}_{x}$ electrodes of the supercapacitors were constructed using lamination (Scheme S1†). The all-solution-processed PSCs constructed at low temperatures exhibited high transparency, great flexibility, and excellent cycling stability, etc., which make them suitable for printing, roll-to-roll manufacturing, and blading/ shearing for making high-efficiency PSCs.

\section{Results and discussion}

\section{Fabrication of the MXene transparent electrodes}

The $\mathrm{Ti}_{3} \mathrm{C}_{2} \mathrm{~T}_{x}$ MXene has shown excellent electronic conductivity and electrochemical performance, which are ideal for fabricating transparent electrodes and powerful transparent supercapacitors. We first prepared the $\mathrm{Ti}_{3} \mathrm{C}_{2} \mathrm{~T}_{x}$ MXene by selective etching of $\mathrm{Al}$ from $\mathrm{Ti}_{3} \mathrm{AlC}_{2}$ MAX phase following the minimally intensive layer delamination (MILD) method (see Fig. 1a and the Experimental section in the ESI $\dagger$ ). ${ }^{33}$ This protocol offers delamination of the etched powder to form a colloidal solution of $\mathrm{Ti}_{3} \mathrm{C}_{2} \mathrm{~T}_{x}$ flakes via manual shaking in deionized water. The synthesized $\mathrm{Ti}_{3} \mathrm{C}_{2} \mathrm{~T}_{x}$ colloidal dispersion exhibits the Tyndall effect, manifesting inherent colloidal stability caused by negative surface charge (Fig. S1 $\uparrow$ ). The colloidal solution resulting from this method contains $\mathrm{Ti}_{3} \mathrm{C}_{2} \mathrm{~T}_{x}$ single sheets with a lateral size of a few micrometers (Fig. 1b). High-magnification transmission electron microscopy (TEM) images (Fig. 1c) reveal the typical appearance of a single sheet of $\mathrm{Ti}_{3} \mathrm{C}_{2} \mathrm{~T}_{x}$ MXene. ${ }^{34}$ In addition, the thickness of the flakes is around $1.5 \mathrm{~nm}$, according to the atomic force microscopy (AFM) measurements in Fig. 1d, in good agreement with a previous report. ${ }^{35}$ Furthermore, the sizes of the $\mathrm{Ti}_{3} \mathrm{C}_{2} \mathrm{~T}_{x}$ flakes can be reduced to smaller than $0.5 \mu \mathrm{m}$ by probe sonication, as shown in the scanning electron microscope images (SEM, Fig. 1e). The lateral size distribution of the $\mathrm{Ti}_{3} \mathrm{C}_{2} \mathrm{~T}_{x}$ flakes before sonication is concentrated in the range 1-4 $\mu \mathrm{m}$ (Fig. 1f), but the majority of the $\mathrm{Ti}_{3} \mathrm{C}_{2} \mathrm{~T}_{x}$ flakes after sonication are smaller than $1 \mu \mathrm{m}$ (Fig. 1g), which is consistent with dynamic light scattering (DLS, Fig. S1c†).

The surface functional groups of MXene make it hydrophilic and enable the solution processability of MXene to obtain highly conductive thin films through a variety of techniques, including vacuum assisted filtration, and spray, spin, and dip coating. ${ }^{36-38}$ In order to meet the requirements of transparent electrodes for OPVs, small-sized MXene flakes obtained by spincoating were chosen for the current study, which could yield smoother, flexible transparent films on any substrate (Fig. 2a, S2 and S3 $\dagger$ ). The two-dimensional GIWAXS patterns of the $\mathrm{Ti}_{3} \mathrm{C}_{2} \mathrm{~T}_{x}$ films prepared by spin-coating exhibit visible arcs of diffracted intensity (Fig. 2b), implying the formation of regular orientations. One-dimensional intensity distribution curves, produced by integrating along the horizontal and vertical directions, were used to investigate the textured stacking structures in detail. The out-of-plane diffraction signals at $q=$ $0.55 \AA^{-1}$ (corresponding to layer spacings of $11.6 \AA$, and also consistent with the interlayer distances of $\mathrm{Ti}_{3} \mathrm{C}_{2} \mathrm{~T}_{x}$ flakes) are significantly stronger than the in-plane signals, suggesting the high degree of alignment of the flakes parallel to the substrate plane under the effect of the shear force. ${ }^{39,40}$ The smooth films produced by this parallel-aligned stacking are a necessary condition for $\mathrm{Ti}_{3} \mathrm{C}_{2} \mathrm{~T}_{x}$ as electrodes of OPVs.

To understand the optoelectronic properties of the $\mathrm{Ti}_{3} \mathrm{C}_{2} \mathrm{~T}_{x}$, the evolution of the transmittance spectra with the thickness of films was measured (Fig. 2d). The spectra show a broad peak in the visible region and the transmittance decreases as the films become thicker. The transmittance at $550 \mathrm{~nm}$ and the conductivity of the spin-coated $\mathrm{Ti}_{3} \mathrm{C}_{2} \mathrm{~T}_{x}$ films as a function of film thickness were plotted and are shown in Fig. 2e. The high transmittance coupled with extraordinary conductivity give $\mathrm{Ti}_{3} \mathrm{C}_{2} \mathrm{~T}_{x}$ excellent optoelectronic properties compared to most other transparent conductive materials. For example, at $\mathrm{T}=$ $86 \%$, the conductivity of $\mathrm{Ti}_{3} \mathrm{C}_{2} \mathrm{~T}_{x}\left(3352 \mathrm{~S} \mathrm{~cm}^{-1}\right)$ is much higher than that of PEDOT:PSS (500 $\left.\mathrm{S} \mathrm{cm})^{-1}\right)^{41}$ or P3-SWCNT $\left(263 \mathrm{~S} \mathrm{~cm}^{-1}\right){ }^{42}$ In addition to outstanding optoelectronic performances in the visible range, the $\mathrm{Ti}_{3} \mathrm{C}_{2} \mathrm{~T}_{x}$ films possess 


\section{(a)}
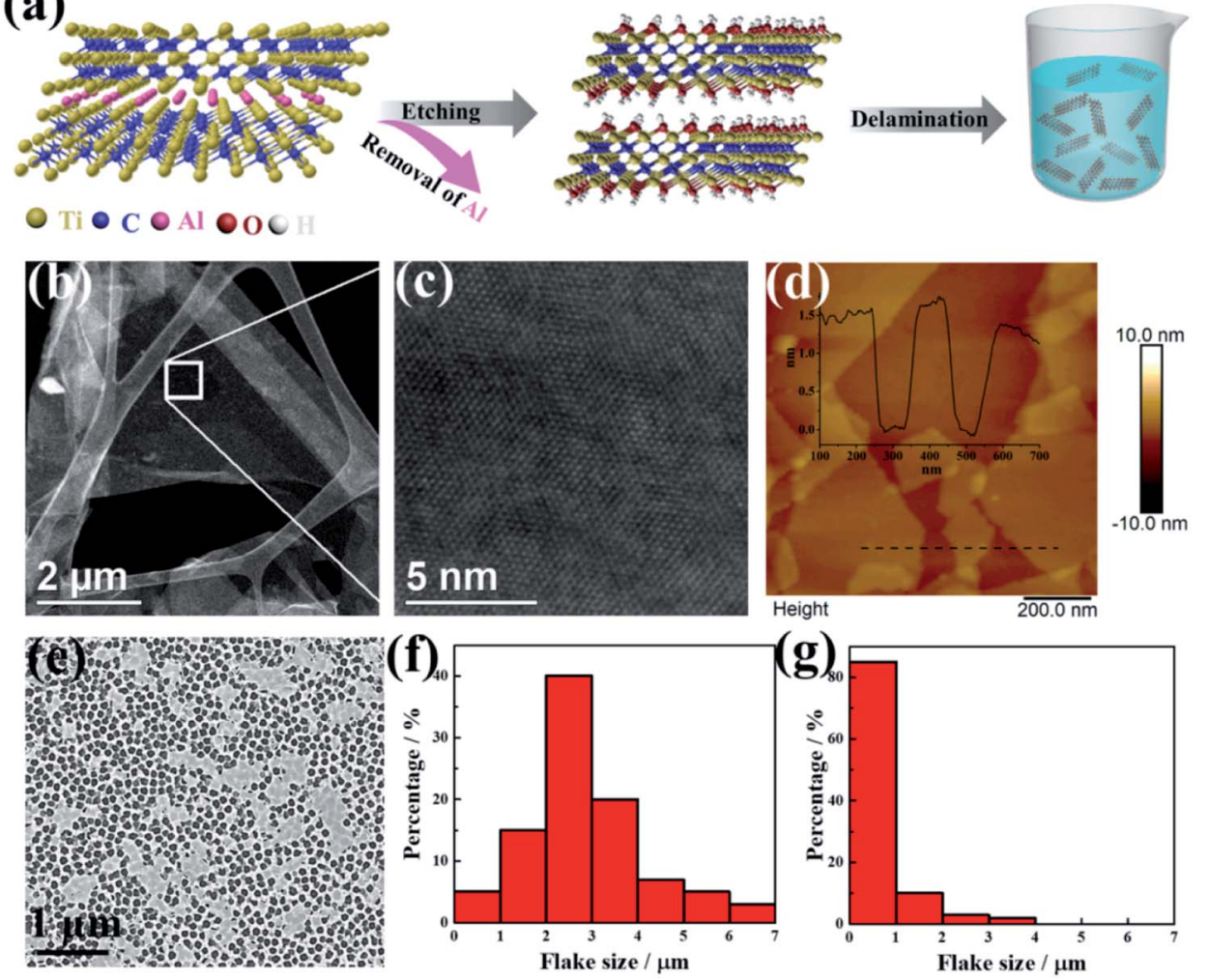

Fig. 1 Fabrication of the $\mathrm{Ti}_{3} \mathrm{C}_{2} \mathrm{~T}_{x}$ MXene colloidal solution. (a) The MXene prepared from the Ti $\mathrm{AlC}_{3} \mathrm{Cl}_{2}$ precursor using the MILD method. (b) Lowmagnification and (c) high-magnification scanning transmission electron microscopy of single $\mathrm{Ti}_{3} \mathrm{C}_{2} \mathrm{~T}_{x}$ sheets dispersed onto a lacy carbon grid. (d) AFM image of $\mathrm{Ti}_{3} \mathrm{C}_{2} T_{x}$ single layer. (e) Top view SEM image of small sized $\mathrm{Ti}_{3} \mathrm{C}_{2} \mathrm{~T}_{x}$. The $\mathrm{Ti}_{3} \mathrm{C}_{2} \mathrm{~T}_{x}$ flake size distributions before (f) and after (g) sonication.

other important advantages: namely, good mechanical flexibility and durability under bending stress. The conductivity of the electrodes on the flexible PET substrates was measured as a function of bending cycles (Fig. 2f). The $\mathrm{Ti}_{3} \mathrm{C}_{2} \mathrm{~T}_{x}$ film exhibits an excellent mechanical stability with a nearly constant resistance after continuous bending cycles, whereas the resistance of the ITO electrode is drastically increased. ${ }^{19} \mathrm{The}^{\mathrm{Ti}} \mathrm{C}_{2} \mathrm{~T}_{x}$ films with high transmittance, conductivity, and excellent mechanical stability motivated us to explore the MXene films as transparent flexible electrodes for high-performance all-solutionprocessed flexible OPVs.

\section{MXene electrode-based OPVs}

Apart from the excellent optical and electrical characteristics of the $\mathrm{Ti}_{3} \mathrm{C}_{2} \mathrm{~T}_{x}$ films, the work function (WF) of the MXene film can be easily modified, which is a very important condition for electrode materials. Here, ultraviolet photoelectron spectroscopy (UPS) was used to probe the WF values of as-caste $\mathrm{Ti}_{3} \mathrm{C}_{2} \mathrm{~T}_{x}$ film and $\mathrm{Ti}_{3} \mathrm{C}_{2} \mathrm{~T}_{x}$ film modified with poly(3,4ethylenedioxythiophene):poly-(styrenesulfonate) (PEDOT:PSS) or polyethylenimine (PEI), as shown in Fig. 3a. From the position of the secondary electron cut-off, the spectra reveal that the WF of $\mathrm{Ti}_{3} \mathrm{C}_{2} \mathrm{~T}_{x}$ is reduced from 4.36 to $3.62 \mathrm{eV}$ after PEI modification, while the WF increases from 4.63 to $5.03 \mathrm{eV}$ after PEDOT:PSS modification, meaning that the $\mathrm{Ti}_{3} \mathrm{C}_{2} \mathrm{~T}_{x}$ film can be used as both hole and electron collecting electrodes of OPVs, depending on the choice of overlayer.

With the combined high-performance features of $\mathrm{Ti}_{3} \mathrm{C}_{2} \mathrm{~T}_{x}$ films, wider tests and studies were carried out on a series of OPVs with different types of donors and acceptors (PM6, PTB7Th, P3HT, Y6, ${ }^{43} \mathrm{PC}_{71} \mathrm{BM}, \mathrm{ICBA}$ ) in the active layers (Fig. S6 $\dagger$ ), to prove their potential application in flexible organic electronics. The detailed fabrication procedure can be found in the Experimental section. The device architecture and corresponding energy level diagram are presented in Fig. $3 \mathrm{~b}, \mathrm{~S} 7$ and ESI, $\uparrow$ respectively. Based on $\mathrm{Ti}_{3} \mathrm{C}_{2} \mathrm{~T}_{x} /$ glass substrates, we fabricated OPVs with different thicknesses of $\mathrm{Ti}_{3} \mathrm{C}_{2} \mathrm{~T}_{x}$ films. For comparison, control devices with ITO $(\sim 110 \mathrm{~nm}) /$ glass were fabricated. The current density-voltage $(J-V)$ characteristics of the OPVs were measured under standard 1 sun simulated solar illumination using Air Mass 1.5 global (AM 1.5G) conditions and an irradiation intensity of $100 \mathrm{~mW} \mathrm{~cm} \mathrm{~cm}^{-2}$ (Fig. S8 and S9†). All the photovoltaic parameters of the devices are listed in Table S1. $\dagger$ With increasing thickness of $\mathrm{Ti}_{3} \mathrm{C}_{2} \mathrm{~T}_{x}$ film, the power conversion efficiency (PCE) of the device increases at first and then decreases. This should reflect the compromise between conductivity and transmittance. The PCE reaches $13.62 \%$ (for PM6:Y6) and 7.76\% (for PTB7-Th:PC ${ }_{71} \mathrm{BM}$ ) with a $17 \mathrm{~nm} \mathrm{Ti}{ }_{3} \mathrm{C}_{2} \mathrm{~T}_{x}$ film, which are comparable values to those of the control devices with ITO/glass substrates (Fig. 3c). In addition, the 
(a)
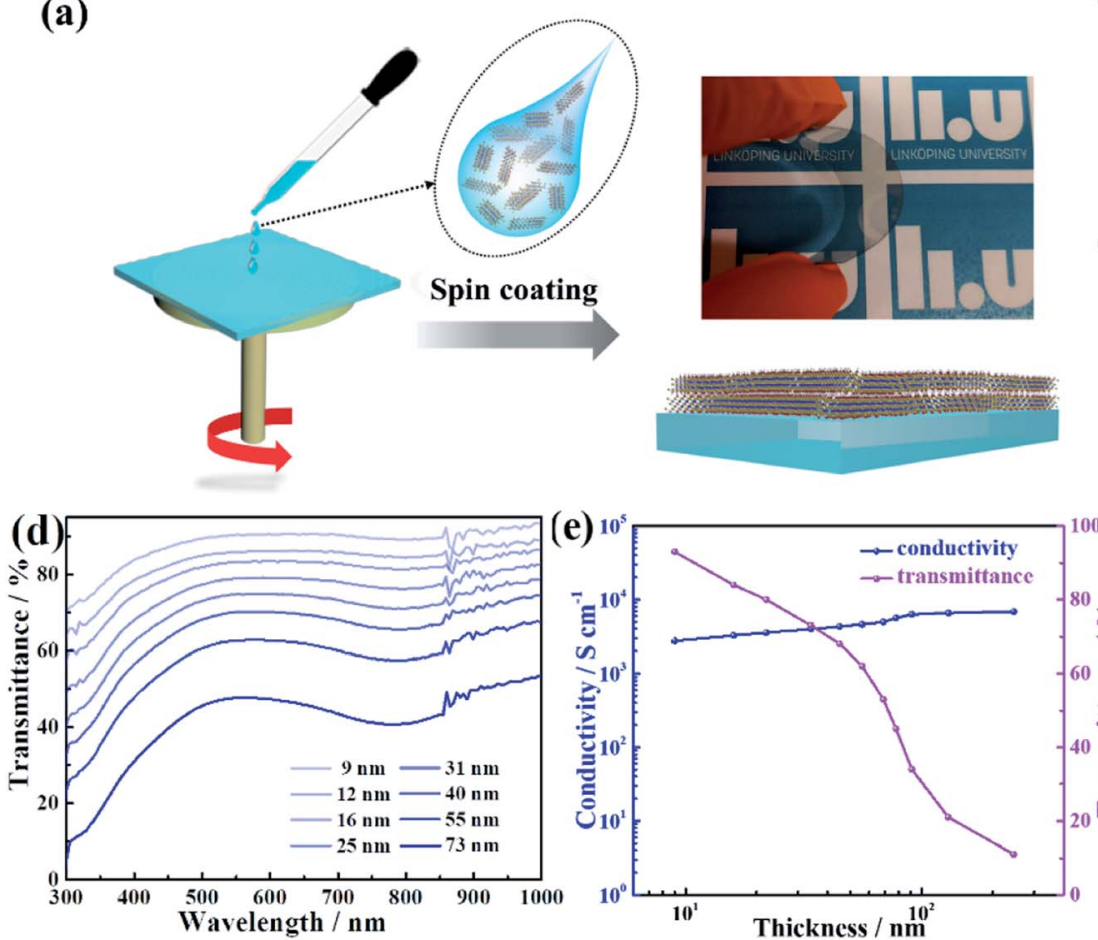

(b)

(c)
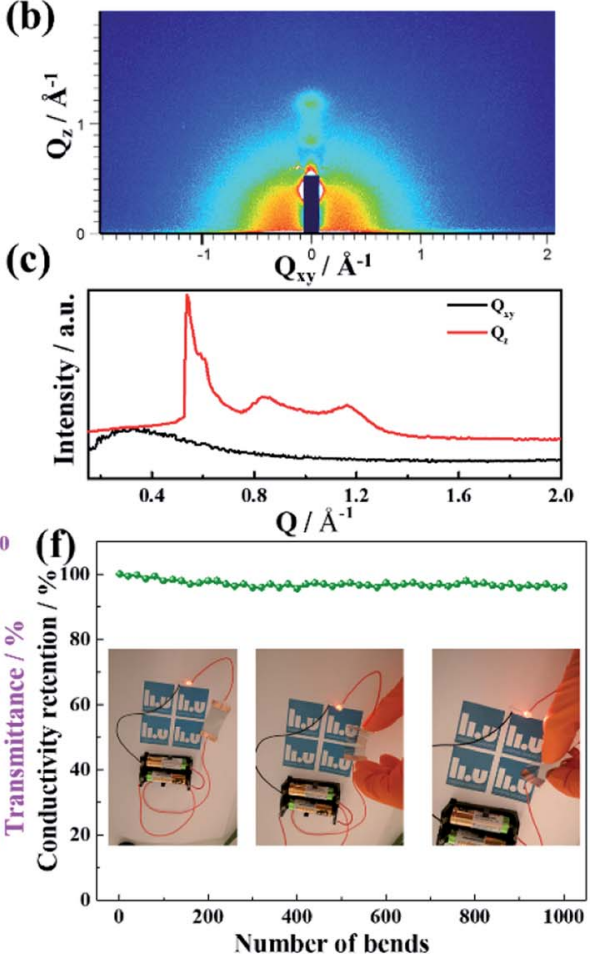

Fig. 2 Optoelectronic properties of $\mathrm{Ti}_{3} \mathrm{C}_{2} \mathrm{~T}_{x}$ films. (a) Schematic of the preparation of a transparent flexible electrode. (b) Two-dimensional GIWAXS pattern of $\mathrm{Ti}_{3} \mathrm{C}_{2} \mathrm{~T}_{x}$ film prepared by the spin-coating method. (c) Out-of-plane and in-plane line-cut profiles from the GIWAXS pattern. (d) Transmittance spectra of $\mathrm{Ti}_{3} \mathrm{C}_{2} \mathrm{~T}_{x}$ films of various thicknesses. (e) Variations in conductivity and transmittance of $\mathrm{Ti}_{3} \mathrm{C}_{2} \mathrm{~T}_{x}$ electrodes as a function of thickness. (f) Conductivity retention with the number of bends for flexible $\mathrm{Ti}_{3} \mathrm{C}_{2} \mathrm{~T}_{x}$ electrodes on PET substrates. The inset image shows the conductivity of the $\mathrm{Ti}_{3} \mathrm{C}_{2} \mathrm{~T}_{x}$ electrodes in bent and twisted states.

flexible devices yield high PCE values of $13.15 \%$ (PM6:Y6) and 7.37\% (PTB7-Th:PC ${ }_{71} \mathrm{BM}$ ) with $\mathrm{Ti}_{3} \mathrm{C}_{2} \mathrm{~T}_{x} / \mathrm{PET}$ substrates. The $\mathrm{Ti}_{3} \mathrm{C}_{2} \mathrm{~T}_{x}$ based devices show slightly lower external quantum efficiency (EQE) compared with ITO based devices in the wavelength range $300-500 \mathrm{~nm}$ (Fig. S10 and S11†), which may be caused by the slightly higher absorption by $\mathrm{Ti}_{3} \mathrm{C}_{2} \mathrm{~T}_{x}$ films at short wavelengths.

To further evaluate the charge collection capability of the $\mathrm{Ti}_{3} \mathrm{C}_{2} \mathrm{~T}_{x}$ MXene transparent electrodes, OPVs with MXene films as both bottom and top electrodes were prepared. In this work, to fabricate semitransparent flexible OPVs by solution processing, a patterned $\mathrm{Ti}_{3} \mathrm{C}_{2} \mathrm{~T}_{x}$ film modified by a thin layer of PEI was used as the bottom electrode. Based on UPS results, PEI modification can significantly reduce the WF of the underlying $\mathrm{Ti}_{3} \mathrm{C}_{2} \mathrm{~T}_{x}$ films and it has been proven to improve the electron collection and performance of various types of solar cells. ${ }^{44,45}$ The poly(3-hexylthiophene) (P3HT):indene-C60 bisadduct (ICBA) or PTB7-Th:PC ${ }_{71} \mathrm{BM}$ and PEDOT:PSS are used as the active layer and hole transport layer, respectively, and are prepared by spin-coating. Finally, the transparent $\mathrm{Ti}_{3} \mathrm{C}_{2} \mathrm{~T}_{x}$ top electrode is prepared by film-transfer lamination. For reference, control devices with ITO $(\sim 110 \mathrm{~nm}) /$ glass were fabricated. The photovoltaic parameters are summarized in Table 1. As shown in Fig. 3d, good efficiency can be achieved when $\mathrm{Ti}_{3} \mathrm{C}_{2} \mathrm{~T}_{x}$ is used as the bottom electrode, top electrode and even as both the top and the bottom electrode for flexible semi-transparent OPVs.
The efficiency is comparable to that of an ITO electrode. Considering that the route to fabrication of $\mathrm{Ti}_{3} \mathrm{C}_{2} \mathrm{~T}_{x}$ based flexible semi-transparent OPVs is facile, cost-effective, and scalable, it is reasonable to assume that $\mathrm{Ti}_{3} \mathrm{C}_{2} \mathrm{~T}_{x}$-based OPVs meet the requirements for energy harvesting units for semitransparent flexible photovoltaic supercapacitors.

\section{Electrochemical performance of transparent MXene supercapacitors}

For semitransparent photovoltaic supercapacitors, not only does the energy conversion part require higher transmittance, but the energy storage part also requires high transmittance. Therefore, high conductivity, high transmission, and excellent capacitance performance are prerequisites for potential candidate materials. $\mathrm{Ti}_{3} \mathrm{C}_{2} \mathrm{~T}_{x}$ MXene has shown significant energystorage capability with excellent flexibility, high conductivity, and optical transparency, suggesting that $\mathrm{Ti}_{3} \mathrm{C}_{2} \mathrm{~T}_{x}$ is suitable for our purpose. ${ }^{28,46,47}$ Considering the choice of electrolyte, a sulfuric acid based electrolyte, which is commonly used for $\mathrm{Ti}_{3} \mathrm{C}_{2} \mathrm{~T}_{x}$ based supercapacitors, can penetrate through the electrode of the capacitor, which would damage the photovoltaic conversion part for vertically integrated energy conversion and storage modules. Based on this consideration, a transparent, flexible solid supercapacitor was constructed using a solid organic ionogel electrolyte instead of a sulfate-based electrolyte (Fig. 4a). 

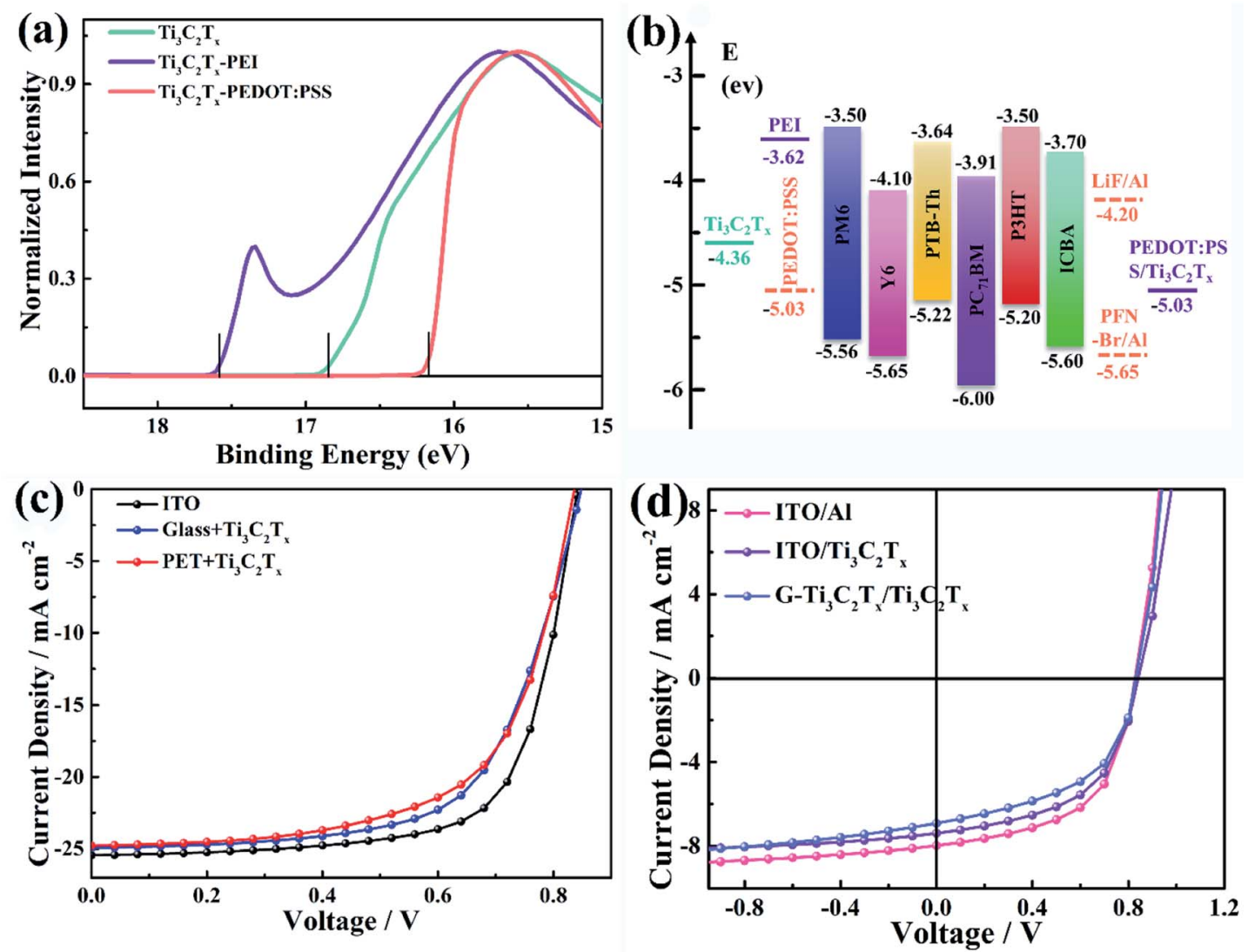

Fig. 3 OPVs performance using MXene transparent electrodes. (a) Ultraviolet photoelectron spectroscopy (UPS) secondary electron cut-off region of $\mathrm{Ti}_{3} \mathrm{C}_{2} \mathrm{~T}_{x}$ and $\mathrm{Ti}_{3} \mathrm{C}_{2} \mathrm{~T}_{x}$ electrodes modified by PEDOT:PSS and PEI. (b) Energy level diagram of OPV devices. (c) $J-V$ curves of OPV devices based on PM6:Y6 with different electrodes. (d) J-V curves of OPV devices based on P3HT:ICBA with different electrodes.

The performance of the transparent, flexible all-solid-state supercapacitor was investigated based on CV curves (Fig. 4b). The shapes of the CV curves are pseudorectangular, even at very high scan rates $\left(500 \mathrm{mV} \mathrm{s}^{-1}\right)$, which indicate highly capacitive behavior and excellent power handling properties in the device. Fig. 4c shows the galvanostatic charging/discharging (GCD) curves of the transparent device. The symmetric sloping shapes of these curves indicate good coulombic efficiency of the charging/discharging process, which is in good agreement with the CV curves shown in Fig. 4b. The volumetric capacitances of the transparent solid-state supercapacitors are calculated from the CVs and GCD profiles and are presented in Fig. 4d. The

Table 1 Photovoltaic parameters of OPVs with different electrodes under AM $1.5 \mathrm{G} 100 \mathrm{~mW} \mathrm{~cm}^{-2}$ illumination $^{a}$

\begin{tabular}{|c|c|c|c|c|c|}
\hline Device configuration & $J_{\mathrm{sc}}\left[\mathrm{mA} \mathrm{cm}^{-2}\right]$ & $V_{\mathrm{oc}}[\mathrm{V}]$ & $\mathrm{FF}[\%]$ & $\operatorname{PCE}^{b}[\%]$ & $\operatorname{AVT}^{c}[\%]$ \\
\hline Glass/ITO/PEDOT:PSS/PM6:Y6/PFN-Br/Al & 25.46 & 0.84 & 70.3 & $15.07(14.97 \pm 0.11)$ & - \\
\hline Glass/ $\mathrm{Ti}_{3} \mathrm{C}_{2} \mathrm{~T}_{x} / \mathrm{PEDOT}: \mathrm{PSS} / \mathrm{PM6}$ :Y6/PFN-Br/Al & 24.97 & 0.84 & 64.9 & $13.62(13.45 \pm 0.21)$ & - \\
\hline PET/Ti ${ }_{3} \mathrm{C}_{2} \mathrm{~T}_{x} / \mathrm{PEDOT}: \mathrm{PSS} / \mathrm{PM6:Y6/PFN-Br/Ag}$ & 24.78 & 0.83 & 0.64 & $13.15(13.01 \pm 0.24)$ & - \\
\hline Glass/ITO/PEDOT:PSS/PTB7-Th:PC ${ }_{71} \mathrm{BM} / \mathrm{LiF} / \mathrm{Al}$ & 14.95 & 0.79 & 69.79 & $8.24(8.11 \pm 0.10)$ & - \\
\hline Glass/ $\mathrm{Ti}_{3} \mathrm{C}_{2} \mathrm{~T}_{x} / \mathrm{PEDOT}: \mathrm{PSS} / \mathrm{PTB} 7-\mathrm{Th}: \mathrm{PC}_{71} \mathrm{BM} / \mathrm{LiF} / \mathrm{Al}$ & 14.91 & 0.79 & 65.61 & $7.76(7.58 \pm 0.12)$ & - \\
\hline PET/Ti ${ }_{3} \mathrm{C}_{2} \mathrm{~T}_{x} / \mathrm{PEDOT}: \mathrm{PSS} / \mathrm{PTB} 7-\mathrm{Th}: \mathrm{PC}_{71} \mathrm{BM} / \mathrm{LiF} / \mathrm{Ag}$ & 14.21 & 0.79 & 65.32 & $7.37(7.13 \pm 0.09)$ & - \\
\hline PET/Ti ${ }_{3} \mathrm{C}_{2} \mathrm{~T}_{x} / \mathrm{PEI} / \mathrm{PTB} 7-\mathrm{Th}: \mathrm{PC}_{71} \mathrm{BM} / \mathrm{PEDOT}: \mathrm{PSS} / \mathrm{Ti}_{3} \mathrm{C}_{2} \mathrm{~T}_{x}$ & 14.43 & 0.75 & 48.73 & $5.26(5.10 \pm 0.14)$ & 31.7 \\
\hline Glass/ITO/PEI/P3HT:ICBA/MoO $/$ /Al & 7.98 & 0.83 & 56.05 & $3.70(3.42 \pm 0.21)$ & - \\
\hline Glass/ITO/PEI/P3HT:ICBA/PEDOT:PSS/Ti ${ }_{3} \mathrm{C}_{2} \mathrm{~T}_{x}$ & 7.39 & 0.84 & 53.71 & $3.34(3.19 \pm 0.11)$ & 51.7 \\
\hline Glass/ $\mathrm{Ti}_{3} \mathrm{C}_{2} \mathrm{~T}_{x} / \mathrm{PEI} / \mathrm{P} 3 \mathrm{HT}: \mathrm{ICBA} / \mathrm{PEDOT}: \mathrm{PSS} / \mathrm{Ti}_{3} \mathrm{C}_{2} \mathrm{~T}_{x}$ & 6.91 & 0.84 & 51.53 & $2.96(2.75 \pm 0.19)$ & 42.3 \\
\hline PET/ $\mathrm{Ti}_{3} \mathrm{C}_{2} \mathrm{~T}_{x} / \mathrm{PEI} / \mathrm{P} 3 \mathrm{HT}: \mathrm{ICBA} / \mathrm{PEDOT}: \mathrm{PSS} / \mathrm{Ti}_{3} \mathrm{C}_{2} \mathrm{~T}_{x}$ & 6.28 & 0.82 & 50.84 & $2.62(2.41 \pm 0.12)$ & 41.5 \\
\hline \multirow{2}{*}{ PET/Ti ${ }_{3} \mathrm{C}_{2} \mathrm{~T}_{x} / \mathrm{PEI} / \mathrm{P} 3 \mathrm{HT}: I C B A / P E D O T: P S S / \mathrm{Ti}_{3} \mathrm{C}_{2} \mathrm{~T}_{x} /$ ionogel/ $/ \mathrm{Ti}_{3} \mathrm{C}_{2} \mathrm{~T}_{x} / \mathrm{PDMS}$} & $6.89^{d}$ & 0.80 & 45.32 & $2.50(2.21 \pm 0.22)$ & 33.5 \\
\hline & $6.45^{e}$ & 0.80 & 36.59 & $1.89(1.73 \pm 0.13)$ & 33.5 \\
\hline
\end{tabular}

${ }^{a} J_{\mathrm{sc}}$, short-circuit current density; $V_{\mathrm{oc}}$, open-circuit voltage; FF, fill factor. ${ }^{b}$ Average PCEs are based on 20 independent devices. ${ }^{c}$ AVT is the average visible transmittance in the range $380-780 \mathrm{~nm} .{ }^{d}$ Photovoltaic performance of a flexible PSC with light from the OPV direction. ${ }^{e}$ Photovoltaic performance of flexible PSC with light from the SC direction. 
(a)
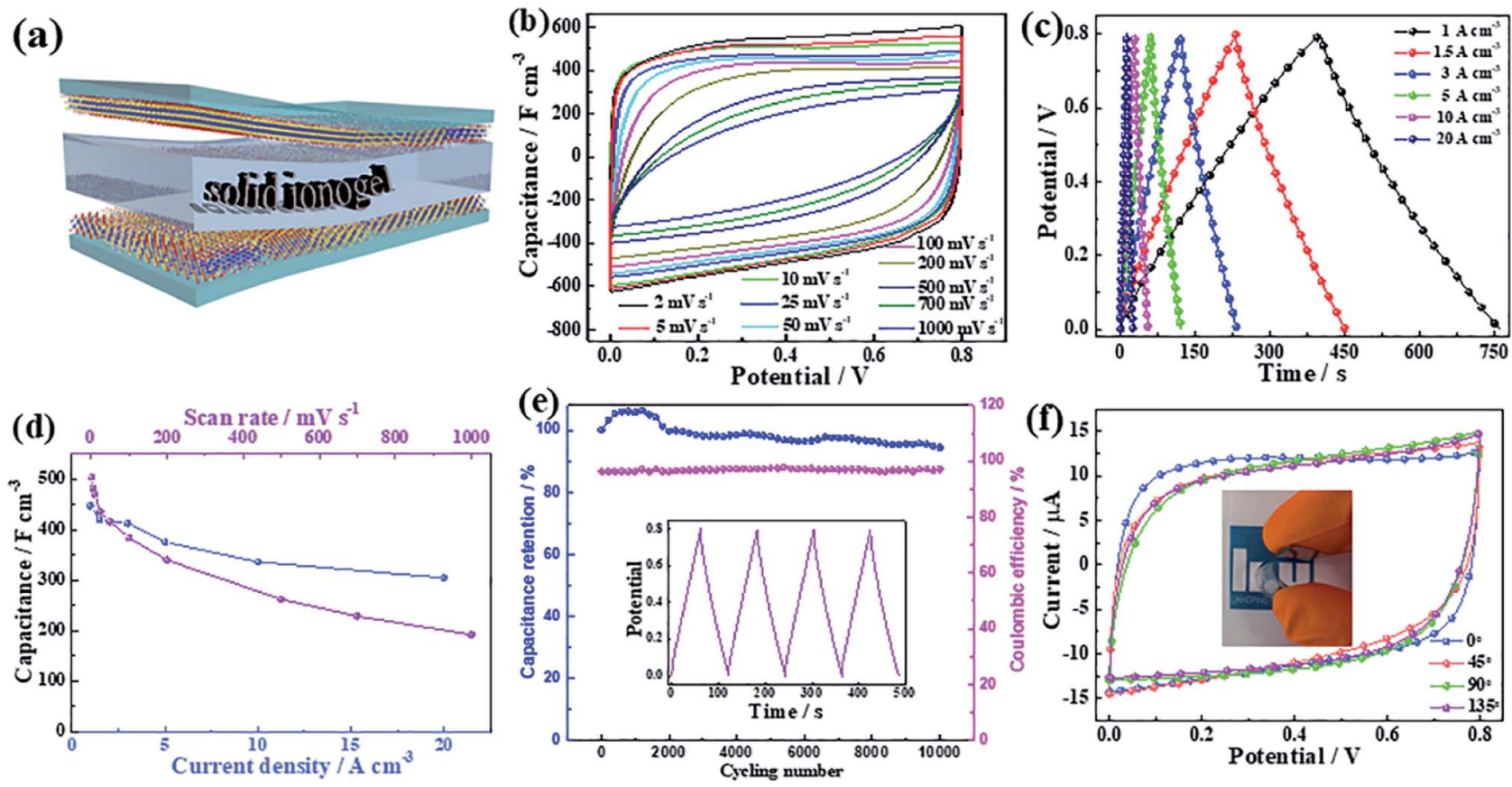

Fig. 4 Electrochemical performances of transparent, flexible, solid-state supercapacitors based on $\mathrm{Ti}_{3} \mathrm{C}_{2} \mathrm{~T}_{x}$ films. (a) Schematic illustration of a flexible solid-state supercapacitor based on spin coating $\mathrm{Ti}_{3} \mathrm{C}_{2} \mathrm{~T}_{x}$ MXene on PET sheets as electrodes and solid ionogel as the electrolyte. (b) $\mathrm{CV}$ curves at various scan rates and (c) galvanostatic charge/discharge (GCD) curves at different current densities for the flexible solid-state device. (d) The volumetric capacitances obtained from GCD and CV. (e) Long-term charge-discharge cycling performance and coulombic efficiency of the $\mathrm{Ti}_{3} \mathrm{C}_{2} \mathrm{~T}_{x}$ based flexible solid-state device. Inset shows the typical GCD curves upon cycling. (f) $\mathrm{CV}$ curves of the $\mathrm{Ti}_{3} \mathrm{C}_{2} \mathrm{~T}_{x}$ device bent to different angles at $50 \mathrm{mV} \mathrm{s}^{-1}$. Inset: optical image of the transparent flexible ultra-thin supercapacitor.

capacitance reach $450 \mathrm{~F} \mathrm{~cm}^{-3}$ at $1 \mathrm{~A} \mathrm{~cm}^{-3}\left(502 \mathrm{~F} \mathrm{~cm}^{-3}\right.$ at $2 \mathrm{mV}$ $\mathrm{s}^{-1}$ ) and maintains $68 \%$ of initial capacitance as the current density is increased by 20 times, indicating the high-rate capability of supercapacitors based on an MXene electrode and an organic ionogel electrolyte, which is comparable with that of a sulfuric acid based electrolyte. ${ }^{28}$ To show further the merits of supercapacitors based on an MXene electrode and an organic ionogel electrolyte, the electrochemical impedance spectra (EIS) of the devices are shown in Fig. S17. $\dagger$ The curve shows a large slope close to $90^{\circ}$ at the low frequency of the complex plane plots, which indicates fast ion diffusion between the organic ionogel electrolyte and the $\mathrm{Ti}_{3} \mathrm{C}_{2} \mathrm{~T}_{x}$ thin films. At the high frequency, see inset, it shows a small charge-transport semicircle, which is consistent with the high conductivity of the $\mathrm{Ti}_{3} \mathrm{C}_{2} \mathrm{~T}_{x}$ films. The lifetime of the flexible transparent device was evaluated through GCD measurements (Fig. 4e). 95\% of initial capacitance was retained after 10000 cycles. In addition, the device also exhibits high stability under different bending angles (Fig. 4f). The high volumetric capacitances, long lifetime, and high transmittance make the organic ionogel electrolyte based $\mathrm{Ti}_{3} \mathrm{C}_{2} \mathrm{~T}_{x}$ flexible supercapacitor the best choice for the energy storage modules of flexible semi-transparent photovoltaic supercapacitors.

\section{The performance of PSCs}

In order to achieve simultaneous energy harvesting and storage in a single device, a flexible semi-transparent photovoltaic supercapacitor was fabricated by vertically integrating energy harvesting and energy storage units by employing $\mathrm{Ti}_{3} \mathrm{C}_{2} \mathrm{~T}_{x}$ transparent thin film as a common electrode. The procedure is briefly summarized as follows (more details in the Experimental section, ESI $\dot{\dagger}$ ): based on the transparent flexible energy harvesting part, the organic ionogel electrolyte and $\mathrm{Ti}_{3} \mathrm{C}_{2} \mathrm{~T}_{x}$ thin films are used as the separator and active electrode of supercapacitor, respectively, and laminated on the transparent top electrode of the OPV to form the energy storage device. This means that the middle $\mathrm{Ti}_{3} \mathrm{C}_{2} \mathrm{~T}_{x}$ thin layer acts not only as the top electrode for the OPV, but also acts as the active electrode for the supercapacitor. As shown in Fig. 5a, the transmittance spectra of the single electrode and the symmetric device show that the transmittance of the single electrode is around $90 \%$ and $70 \%$ can be achieved for a device, which is higher than the transmittance of a reported supercapacitor. ${ }^{28}$ The average visible transmittance (AVT) of the flexible transparent OPV is $42 \%$. Finally, the flexible transparent PSC exhibits an AVT of over $33.5 \%$. Fig. $5 \mathrm{~b}$ shows the $J-V$ characteristics of a flexible transparent PSC illuminated from the OPV and the supercapacitor side, respectively. When illuminated from the OPV device, the PESM shows $V_{\text {oc }}=0.81 \mathrm{~V}, J_{\mathrm{sc}}=6.89 \mathrm{~mA} \mathrm{~cm}^{-2}$, and $F$ $=0.45$, yielding an average PCE of $2.5 \%$. When illuminated from the supercapacitor device, a lower $J_{\mathrm{sc}}$ value is obtained due to the lower transmittance of the top energy storage device. The photocharging and discharging principle (Fig. S19†) and performance (Fig. 5c) of the PSC were investigated under an illumination of AM $1.5\left(100 \mathrm{~mW} \mathrm{~cm}^{-2}\right)$. In the charging process 

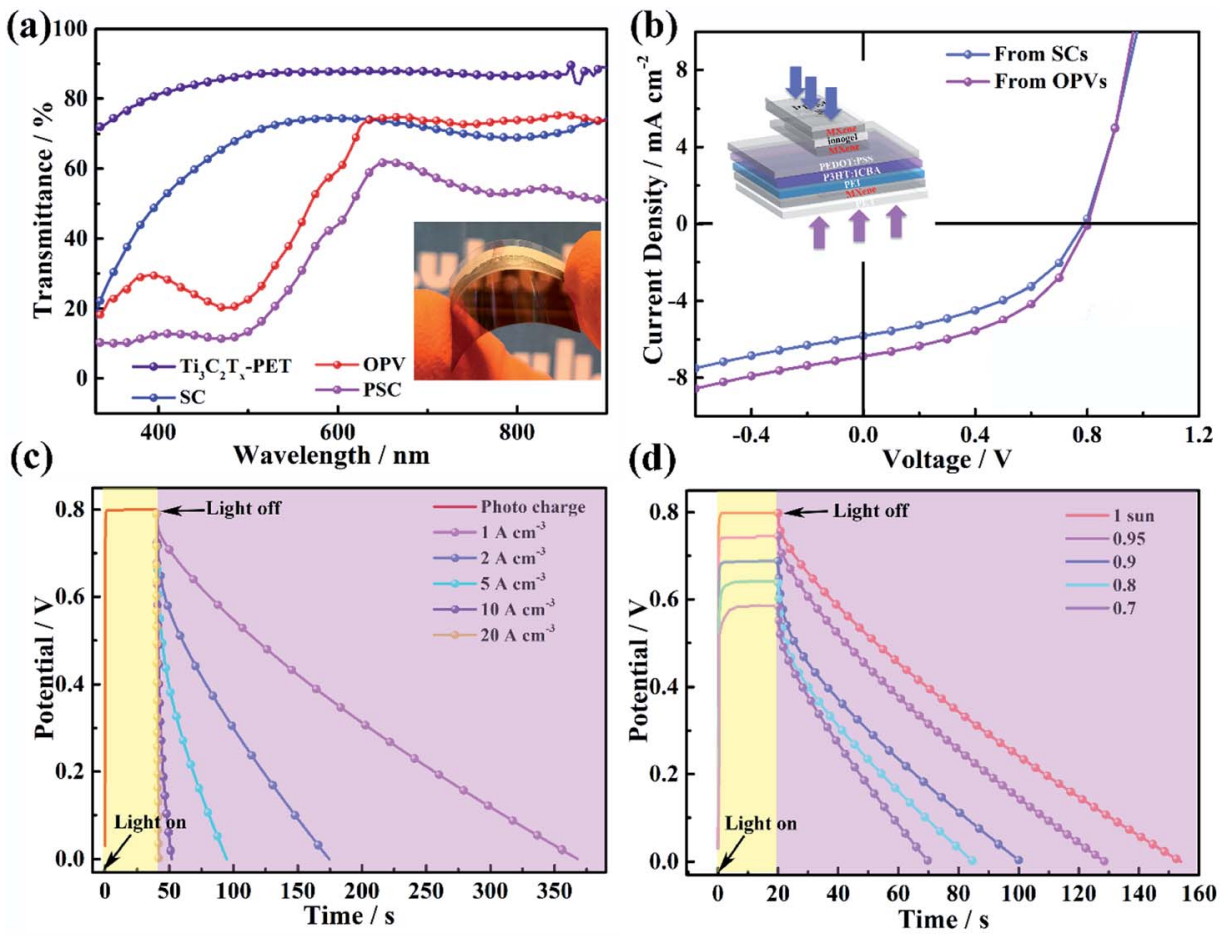

Fig. 5 The performance of PSCs based on MXene electrodes. (a) Transmittance spectra of a single transparent $\mathrm{Ti}_{3} \mathrm{C}_{2} \mathrm{~T}_{x}$ electrode, transparent supercapacitors based on $\mathrm{Ti}_{3} \mathrm{C}_{2} \mathrm{~T}_{x}$ films, a transparent OPV with P3HT:ICBA as active layer and a transparent, flexible PSC. Inset: a photograph of a transparent, flexible OPV device based on $\mathrm{Ti}_{3} \mathrm{C}_{2} \mathrm{~T}_{x}$ electrodes; the red circle represents the OPV device part. (b) $\mathrm{J}-\mathrm{V}$ characteristics of allsolution-processed flexible PSCs with different light source directions. Inset, top: the device structure of the PSC; bottom: a photograph of a transparent, flexible PSC device. (c) The voltage transients of the capacitor during photo-charging under AM 1.5 simulated sunlight illumination, and galvanostatic discharge at different discharge current densities. (d) Photo charge of PSC with different light irradiance and galvanostatic discharge at $2 \mathrm{~A} \mathrm{~cm}^{-3}$

(yellow section), the PSC reaches a capacitor voltage $\left(V_{\text {cap }}\right)$ of $0.8 \mathrm{~V}$ within a very short charging time of $2 \mathrm{~s}$. Under continuous illumination, the $V_{\text {cap }}$ value remains the same at the saturated values of $0.8 \mathrm{~V}$. The discharge behavior of the PSCs under different current densities after shutting down the light source was evaluated in Fig. 5c (purple section). The specific capacitance at a discharge current density of $1 \mathrm{~A} \mathrm{~cm}^{-3}$ exhibits $410 \mathrm{~F}$ $\mathrm{cm}^{-3}$, which is close to the maximum performance of an unintegrated $\mathrm{Ti}_{3} \mathrm{C}_{2} \mathrm{~T}_{x}$-based flexible transparent supercapacitor, indicating the successful integration of the energy storage unit into the energy harvesting device. Furthermore, the photo charging and discharging performance of the PSC were evaluated as a function of light irradiance. As shown in Fig. 5d, with decreasing light intensity, the photo charging time increases and the light saturated $V_{\text {cap }}$ decreases. This is due to the decrease in $J_{\mathrm{sc}}$ and $V_{\mathrm{oc}}$ at low irradiance in the energy harvesting device. The overall photoelectric conversion and storage efficiency versus photocharging time were calculated. As shown in Fig. S20,$\dagger$ a self-power pack with a flexible semi-transparent PSC had maximum $\eta_{\text {storage }}$ and $\eta_{\text {overall }}$ values of up to $88 \%$ and $2.2 \%$, respectively. Considering that the efficiency of the energy storage module is high, the overall efficiency is strongly dependent on the energy harvesting part. Further optimization can be expected by utilizing efficient and stable organic photovoltaic materials.

\section{Conclusions}

In summary, MXene-based all-solution-processed flexible semitransparent PSCs were fabricated with a vertically integrated $\mathrm{Ti}_{3} \mathrm{C}_{2} \mathrm{~T}_{x}$-electrode-based transparent flexible photovoltaic device and $\mathrm{Ti}_{3} \mathrm{C}_{2} \mathrm{~T}_{x}$-based transparent supercapacitor. $\mathrm{Ti}_{3} \mathrm{C}_{2} \mathrm{~T}_{x}$ transparent films were produced, aligned parallel to the substrate and demonstrating outstanding flexibility, transmittance, and conductivity. These properties make the films efficient transparent electrodes for OPV with an obtained high PCE of 13.6\%, comparable with that of ITO electrodes, and as the active material for supercapacitors with a high volumetric capacitance of $502 \mathrm{~F} \mathrm{~cm}^{-3}$. The long cycling stability is based on a highperformance organic solid ionogel electrolyte. The organic solid electrolyte effectively solves the issue of the leakage of water-based electrolyte from the energy storage device for the energy harvesting device during vertical integration. Finally, the semi-transparent flexible PSC with $\mathrm{Ti}_{3} \mathrm{C}_{2} \mathrm{~T}_{x}$ thin films as the common electrodes exhibits an AVT of $33.5 \%$, and maximum $\eta_{\text {storage }}$ and $\eta_{\text {overall }}$ values of up to $88 \%$ and $2.2 \%$, respectively. Undoubtedly, through further optimization of the efficiency of the energy harvesting part, the overall efficiency of the PSC devices could be further improved. This all-solution-processing semitransparent flexible PSC is suitable for blading, printing, and roll-to-roll manufacturing, which is promising for the 
production of cost-efficient flexible PSCs to satisfy the increasing energy demands for portable, wearable and miniature electronic devices.

\section{Conflicts of interest}

There are no conflicts of interest to declare.

\section{Acknowledgements}

This work was financed by the Swedish Energy Agency (EM 42033-1), the Swedish Government Strategic Research Area in Material Science on Functional Materials at Linköping University (Faculty Grant SFO-Mat-LiU No 200900971) and the Swedish Research Council (2017-04123), the SSF Research Infrastructure Fellow Program no. RIF 14-0074 and the SSF Synergy Program EM16-0004, and by the Knut and Alice Wallenberg (KAW) Foundation through a Fellowship Grant, a Project Grant (KAW 2015.0043), and for support of the electron microscopy laboratory and the device physics lab in Linköping. Support from the National Natural Science Foundation of China (61774077), the Open Fund of the State Key Laboratory of Luminescent Materials and Devices (2018-skllmd-12) and the Fundamental Research Funds for the Central Universities are also acknowledged.

\section{References}

1 S. Chu and A. Majumdar, Nature, 2012, 488, 294-303.

2 Y. Fu, H. Wu, S. Ye, X. Cai, X. Yu, S. Hou, H. Kafafy and D. Zou, Energy Environ. Sci., 2013, 6, 805-812.

3 S. Park, S. W. Heo, W. Lee, D. Inoue, Z. Jiang, K. Yu, H. Jinno, D. Hashizume, M. Sekino, T. Yokota, K. Fukuda, K. Tajima and T. Someya, Nature, 2018, 561, 516-521.

4 G. Li, R. Zhu and Y. Yang, Nat. Photonics, 2012, 6, 153-161. 5 Y. Liu, J. Zhao, Z. Li, C. Mu, W. Ma, H. Hu, K. Jiang, H. Lin, H. Ade and H. Yan, Nat. Commun., 2014, 5, 5293.

6 Z. He, B. Xiao, F. Liu, H. Wu, Y. Yang, S. Xiao, C. Wang, T. P. Russell and C. Yong, Nat. Photonics, 2015, 9, 174-179.

7 Y.-B. Cheng, A. Pascoe, F. Huang and Y. Peng, Nature, 2016, 539, 488-489.

8 F. Zhang, M. Johansson, M. R. Andersson, J. C. Hummelen and O. Inganäs, Adv. Mater., 2002, 14, 662-665.

9 J. L. Smith, Science, 1981, 212, 1472-1478.

10 Y. Jin, Z. Li, L. Qin, X. Liu, L. Mao, Y. Wang, F. Qin, Y. Liu, Y. Zhou and F. Zhang, Adv. Mater., 2017, 4, 1700704.

11 G. Wee, T. Salim, Y. M. Lam, S. G. Mhaisalkar and M. Srinivasan, Energy Environ. Sci., 2011, 4, 413-416.

12 S. Pan, J. Ren, X. Fang and H. Peng, Adv. Energy Mater., 2016, 6, 1501867.

13 Z. Zhang, X. Chen, P. Chen, G. Guan, L. Qiu, H. Lin, Z. Yang, W. Bai, Y. Lou and H. Peng, Adv. Mater., 2014, 26, 466-470.

14 W. Guo, X. Xue, S. Wang, C. Lin and Z. Wang, Nano Lett., 2012, 12, 2520-2523.

15 J. Xu, H. Wu, S. Wang, C. Lin and Z. Wang, Adv. Funct. Mater., 2013, 24, 1840-1846.
16 K. Nomura, H. Ohta, A. Takagi, T. Kamiya, M. Hirano and H. Hosono, Nature, 2004, 432, 488-492.

17 T. Georgiou, R. Jalil, B. D. Belle, L. Britnell, R. V. Gorbachev, S. V. Morozov, Y.-J. Kim, A. Gholinia, S. J. Haigh and O. Makarovsky, Nat. Nanotechnol., 2013, 8, 100-103.

18 H. Kang, G. Kim, J. Kim, S. Kwon, H. Kim and K. Lee, Adv. Mater., 2016, 28, 7821-7861.

19 H. Kang, S. Jung, S. Jeong, G. Kim and K. Lee, Nat. Commun., 2015, 6, 6503.

20 S. Ye, A. R. Rathmell, Z. Chen, I. E. Stewart and B. J. Wiley, Adv. Mater., 2014, 26, 6670-6687.

21 N. Espinosa, R. Garcia-Valverde and F. C. Krebs, Energy Environ. Sci., 2011, 4, 1547-1557.

22 M. Naguib, M. Kurtoglu, V. Presser, J. Lu, J. Niu, M. Heon, L. Hultman, Y. Gogotsi and M. W. Barsoum, Adv. Mater., 2011, 23, 4248-4253.

23 B. Anasori, M. R. Lukatskaya and Y. Gogotsi, Nat. Rev. Mater., 2017, 2, 16098.

24 M. Ghidiu, M. R. Lukatskaya, M.-Q. Zhao, Y. Gogotsi and M. W. Barsoum, Nature, 2014, 516, 78-81.

25 L. Qin, Q. Tao, X. Liu, M. Fahlman, J. Halim, P. Persson, J. Rosen and F. Zhang, Nano Energy, 2019, 60, 734-742.

26 M. R. Lukatskaya, O. Mashtalir, C. E. Ren, Y. Dall'Agness, P. Rozier, P. L. Taberna, M. Naguib, P. Simon, M. W. Barsoum and Y. Gogotsi, Science, 2013, 341, 15021505.

27 Y. Xia, T. S. Mathis, M.-Q. Zhao, B. Anasori, A. Dang, Z. Zhou, H. Cho, Y. Gogotsi and S. Yang, Nature, 2018, 557, 409-412.

28 C. Zhang, B. Anasori, A. Seral-Ascaso, S.-H. Park, N. McEvoy, A. Shmeliov, G. S. Duesberg, J. N. Coleman, Y. Gogotsi and V. Nicolosi, Adv. Mater., 2017, 29, 1702678.

29 A. D. Dillon, M. J. Ghidiu, A. L. Krick, J. Griggs, S. J. May, Y. Gogotsi, M. W. Barsoum and A. T. Fafarman, Adv. Funct. Mater., 2016, 26, 4162-4168.

30 C. Zhang, S.-H. Park, A. Seral-Ascaso, S. Barwich, N. McEvoy, C. S. Boland, J. N. Coleman, Y. Gogotsi and V. Nicolosi, High capacity silicon anodes enabled by MXene viscous aqueous ink, Nat. Commun., 2019, 10, 849.

31 C. Zhang, L. McKeon, M. P. Kremer, S.-H. Park, O. Ronan, A. Seral-Ascaso, S. Barwich, C. O. Coileain, N. McEvoy, H. C. Nerl, B. Anasori, J. N. Coleman, Y. Gogotsi and V. Niclolsi, Nat. Commun., 2019, 10, 1795.

32 G. S. Gund, J. H. Park, R. Harpalsinh, M. Kota, J. H. Shin, T. Kim, Y. Gogotsi and H. S. Park, Joule, 2019, 3, 1-13.

33 M. Alhabeb, K. Maleski, B. Anasori, P. Lelyukh, L. Clark, S. Sin and Y. Gogotsi, Chem. Mater., 2017, 29, 7633-7644.

34 L. H. Karlsson, J. Birch, J. Halim, M. W. Barsoum and P. O. Å. Persson, Nano Lett., 2015, 15, 4955-4960.

35 W. Tian, A. VahidMohammadi, Z. Wang, L. Ouyang, M. Beidaghi and M. M. Hamedi, Nat. Commun., 2019, 10, 2558.

36 Q. Jiang, N. Kurra, M. Alhabeb, Y. Gogotsi and H. N. Alshareef, Adv. Energy Mater., 2018, 8, 1703043.

37 P. Salles, E. Quain, N. Kurra, A. Sarycheva and Y. Gogotsi, Small, 2018, 14, 1802864. 
38 Y. Peng, B. Akuzum, N. Kurra, M.-Q. Zhao, M. Alhabeb, B. Anasori, E. C. Kumbur, H. N. Alshareef, M. DerGer and Y. Gogotsi, Energy Environ. Sci., 2016, 9, 2847-2854.

39 A. D. Dillon, M. J. Ghidiu, A. L. Krick, J. Griggs, S. J. May, Y. Gogotsi, M. W. Barsoum and A. T. Fafarman, Adv. Funct. Mater., 2016, 26, 4162-4168.

40 E. Pomerantseva and Y. Gogotsi, Nat. Energy, 2017, 2, 17089. 41 Z. Tang, A. Elfwing, A. Melianas, J. Bergqvist, Q. Bao and O. Inganäs, J. Mater. Chem. A, 2015, 3, 24289-24296.

42 P. J. King, T. M. Higgins, S. De, N. Nicoloso and J. N. Coleman, ACS Nano, 2012, 6, 1732-1741.

43 J. Yuan, Y. Zhang, L. Zhou, G. Zhang, H.-L. Yip, T.-K. Lau, X. Lu, C. Zhu, H. Peng, P. A. Johnson, M. Leclerc, Y. Cao, J. Ulanski, Y. Li and Y. Zou, Joule, 2019, 3, 1140-1151.
44 Y. Zhou, C. Fuentes-Hernandez, J. W. Shim, J. Meyer, A. J. Giordano, H. Li, P. Winget, T. Papadopoulos, H. Cheun, J. Kim, M. Fenoll, A. Dindar, W. Haske, E. Najafabadi, T. M. Khan, H. Sojoudi, S. Barlow, S. Graham, J.-L. Brédas, S. R. Marder, A. Kahn and B. Kippelen, Science, 2012, 336, 327-332.

45 Y. Zhou, C. Fuentes-Hernandez, J. W. Shim, T. M. Khan and B. Kippelen, Energy Environ. Sci., 2012, 5, 9827-9832.

46 M.-Q. Zhao, C. Ren, Z. Ling, M. R. Lukatskaya, C. Zhang, K. L. Van Aken, M. W. Barsoum and Y. Gogotsi, Adv. Mater., 2015, 27, 339-345.

47 M. R. Lukatskaya, S. Kota, Z. Lin, M.-Q. Zhao, N. Shpigel, M. D. Levi, J. Halim, P.-L. Taberna, M. W. Barsoum, P. Simon and Y. Gogotsi, Nat. Energy, 2017, 2, 17105. 